\title{
Gauge-noninvariance of quantum cosmology and vacuum dark energy
}

\author{
Irina Dymnikova \\ Department of Mathematics and Computer Science, University of Warmia and Mazury, \\ Żotnierska 14, 10-561 Olsztyn, Poland; e-mail: irina@matman.uwm.edu.pl \\ Michael Fil'chenkov \\ Alexander Friedmann Laboratory for Theoretical Physics, St. Petersburg, Russia \\ Institute of Gravitation and Cosmology, Peoples' Friendship University of Russia, \\ 6 Miklukho-Maklaya Street, Moscow 117198, Russia; e-mail: fmichael@mail.ru
}

\begin{abstract}
We address the question how to adapt cosmological constant $\Lambda$ for description of a vacuum dark energy density jumping from the big initial value to the small today value suggested by observations. We find such a possibility in the gauge-noninvariance of quantum cosmology which leads to a connection between a choice of the gauge and quantum spectrum for a certain physical quantity which can be specified in the framework of the minisuperspace model. We introduce a particular gauge in which the cosmological constant $\Lambda$ is quantized and show that making a measurement of $\Lambda$ today one can find its small value with the biggest probability, while at the beginning of the evolution, the biggest probability corresponds to its biggest value. Transitions between quantum levels of $\Lambda$ in the course of the Universe evolution, could be related to several scales for symmetry breaking.
\end{abstract}

PACS numbers: 04.70.Bw, 04.20.Dw

Introduction - Astronomical data provide the convincing evidence that our Universe is dominated in $70 \%$ of its density by a dark energy responsible for its accelerated expansion due to negative pressure $p=w \varepsilon ; w<$ $-1 / 3[1]$. Observations constrain the parameter $w$ to $w<-0.7$ with the best fit $w=-1[2,3]$ corresponding to the cosmological constant $\Lambda$ related to a vacuum energy density by $\Lambda=8 \pi G \varepsilon_{v a c}$. But $\Lambda$ is plagued by the problem of cosmological constant: The inflationary paradigm requires it to be large at the earliest evolutionary stages, observations demand that it should be many orders of magnitude less today, while the Einstein equations require it to be constant, and the quantum field theory estimates an upper cutoff on this constant by the Planck scale, the resulting zero-point contribution to gravity is incompatible with all observational data [4].

In this Letter we show the existence of the connection between $\Lambda$ dynamics and the gauge-noninvariance of quantum cosmology which could support cosmological constant $\Lambda$ as a promising dark energy candidate.

In quantum cosmology the whole universe is treated quantum-mechanically and described by a wave function $\Psi$. The wave function of a universe $\Psi\left[h_{i k}(\vec{x}), \phi_{m}(\vec{x})\right]$ is defined on the superspace of all 3-dimensional geometries $h_{i k}(\vec{x})$ and matter field configurations $\phi_{m}(\vec{x})$, and satisfies the Wheeler-DeWitt equation $[5,6]$

$$
\hat{H} \Psi=0 .
$$

In the minisuperspace models for quantum gravity the four-metric is typically written in the Arnowitt-DeserMisner formalism as

$$
d s^{2}=N^{2}(t) d t^{2}-h_{i j} d x^{i} d x^{j},
$$

where $N(t)$ is an arbitrary lapse function.
In quantum geometrodynamics there exists the problem of time gauge [7]. In quantum cosmology the essence of this problem is clearly seen in the minisuperspace examples below.

On the one hand, in the Vilenkin ansatz [8] the four line element is written as

$$
d s^{2}=N^{2}(t) d t^{2}-a^{2}(t) d \Omega_{3}^{2}
$$

where $a(t)$ is the scale factor and $d \Omega_{3}^{2}$ is the metric on a unit 3-sphere. For a homogeneous isotropic and closed model with the cosmological term the action is

$$
S=\frac{1}{16 \pi G} \int d^{4} x \sqrt{-g}[R-2 \Lambda]
$$

and the Lagrangian reads [8]

$$
\mathcal{L}=\frac{1}{2} N\left(a\left[1-\frac{\dot{a}^{2}}{N^{2}}\right]-\Lambda a^{3}\right) .
$$

The canonical form of the Lagrangian is $\mathcal{L}=p_{a} \dot{a}-\mathcal{H}=$ $p_{a} \dot{a}-N(t) H$ where $N(t)$ plays the role of the Lagrange multiplier. The Lagrange equation $\partial \mathcal{L} / \partial N=0$ gives the constraint

$$
H=-\frac{1}{2}\left[\frac{p_{a}^{2}}{a}+a-\Lambda a^{3}\right]=0 .
$$

The Hamiltonian constraint for $H$, related to the Hamiltonian of the dynamical system $\mathcal{H}=N(t) H$, does not involve the time-dependence given by $N(t)$. As a result, the standard quantization procedure $\left(p_{a} \rightarrow-i d / d a\right)$ applied to the constraint (6), gives the Wheeler-DeWittt equation $\hat{H} \Psi=0$ in the form independent of the lapse function $N(t)$ [8] (which is out of configuration space). 
On the other hand, it was emphasized by Halliwell that the Hamiltonian constraint expresses an invariance of the theory under time reparametrization, but the formalism is not invariant under field redefinition involving the lapse function $N(t)[7]$.

Halliwell studied a class of reparametrization-invariant theories described by an action of the form

$$
S=\int d t\left[p_{a} \dot{q}^{a}-N H\left(p_{a}, q^{a}\right)\right]
$$

with a Lagrange multiplier $N$ which enforces the constraint $H=0$. Two minisuperspace models, first with the line element

$$
d s^{2}=-\frac{N^{2}(t)}{q(t)} d t^{2}+q(t) d \Omega_{3}^{2}
$$

and the action (the case $k=0$ )

$$
S=\int d t\left[p \dot{q}-\frac{N}{2}\left(-4 p^{2}-1\right)\right]
$$

and second, with the lapse function $\tilde{N}=q^{-1} N$, the line element

$$
d s^{2}=q(t)\left[\tilde{N}^{2}(t) d t^{2}-d \Omega_{3}^{2}\right]
$$

and the action

$$
S=\int d t\left[p \dot{q}-\frac{\tilde{N}}{2}\left(-4 q p^{2}-q\right)\right]
$$

give two different Wheeler-DeWitt equations and describe different quantum theories, so that the procedure is not invariant under field redifinitions involving the lapse function [7] (see also [9]).

Indeed, as early as in 1967 DeWitt found, in the synchronous gauge $(N=1)$, the energy spectrum of a dustfilled universe in the closed FRW model with a curvaturegenerated potential and without $\Lambda$, which corresponds to quantization in the well with infinite walls [5]. Adding cosmological term $\Lambda g_{\mu \nu}$ results in transformation of an infinite well into a finite barrier [10]. Existence of a finite barrier allows one to consider emergence of a universe in the quantum tunnelling event [11]. In the synchronous gauge a universe would emerge with a certain quantized value of the rest-mass energy. In the conformal gauge $(N=a)$ the quantized quantity taking the role of energy in the WDW equation is related to the contribution of the radiation $(p=\varepsilon / 3)$ to the total energy density [12], which makes possible a quantum birth of a hot universe in the tunnelling event [13].

Here we study consequences of gauge-noninvariance of the theory under field redefinitions involving the lapse function $N$, to $\Lambda$ dynamics.

In quantum cosmology models a universe could pass through stages on which the cosmological constant $\Lambda$ can take different values in a wide range [4]. In particular, a universe could start from a quantum state in which $\Lambda$ had not a certain value; any measurements of the universe properties would give then a distribution of possible values of $\Lambda$ with a priori probabilities defined by an initial state [14].

Following these ideas, we admit that at each stage of its evolution a universe could be found, with a certain probability, in a state with a certain quantum value of $\Lambda$.

We show that the gauge-noninvariance of quantum cosmology leads to a connection between a choice of the gauge function $N$ and quantum spectrum for a certain physical quantity, which we specify in the framework of the minisuperspace model. We introduce a particular gauge in which the cosmological constant $\Lambda$ is quantized. Then the wave function of the universe is $\Psi=\sum_{n} c_{n} \psi_{n}$ which allows one to estimate the probability to find the universe in a state with a certain quantized value $\lambda_{n}$.

Approach - We express the line element in the form

$$
d s^{2}=N^{2}(a) d \eta^{2}-a^{2}(\eta) d \Omega_{3}^{2}
$$

which explicitly takes into account the field redefinitions involving the lapse function. The time-gauge function $N(a)$ enters into the configuration space (as a function of $a$ ), and the dynamical system becomes clearly noninvariant under the gauge transformations $N(a) \rightarrow \tilde{N}(\tilde{a})$.

In fact (12) represents a generalization of the conformal gauge $N(a)=a(d t=a d \eta \rightarrow d t=N(a) d \eta)$.

We start with the action

$$
S=\frac{1}{16 \pi G} \int R \sqrt{-g} d^{4} x .
$$

Using the freedom of adding to the Lagrangian an arbitrary full derivative $d f(\eta) / d \eta$, we express it as

$$
\mathcal{L}=\frac{1}{2} \frac{a \dot{a}^{2}}{N(a)}-\frac{N(a)}{2} a k+\frac{4 \pi G}{3} N(a) \varepsilon(a) a^{3} .
$$

Here dot denotes differentiation with respect to $\eta$, and $k$ is the curvature parameter $(k=-1,0,+1$ for an open, flat and closed model respectively).

The momentum is given by

$$
p_{a}=\frac{a \dot{a}}{N(a)} \text {. }
$$

The Hamiltonian reads

$$
\mathcal{H}=\frac{N(a)}{2} \frac{p_{a}^{2}}{a}+\frac{N(a)}{2} a k-\frac{4 \pi G}{3} N(a) \varepsilon(a) a^{3} .
$$

The canonical form of the Lagrangian is

$$
\mathcal{L}=p_{a} \dot{a}-\mathcal{H}=p_{a} \dot{a}-N(a) \tilde{\mathcal{H}}
$$

where

$$
\tilde{\mathcal{H}}=\frac{1}{2} \frac{p_{a}^{2}}{a}+\frac{1}{2} a k-\frac{4 \pi G}{3} \varepsilon(a) a^{3} .
$$


The gauge function $N(a)$ plays the role of the Lagrange multiplier, which gives the constraint equation

$$
\mathcal{H}=0 .
$$

To present the Hamiltonian (16) in the canonical form, we make the canonic transformation $a, p_{a} \rightarrow q, p_{q}$ such that

$$
p_{q}=\sqrt{\frac{N(a)}{f(a) a}} ; \quad q=\int \sqrt{\frac{a f(a)}{N}} d a
$$

where $f(a)$ is a smooth function. It is easy to check that the second Hamilton equation $\dot{p}_{q}=-\partial \mathcal{H} / \partial q$ is satisfied identically for any function $f(a)$, so we can put $f=1$. The resulting Hamiltonian reads

$$
\mathcal{H}=\frac{p_{q}^{2}}{2}+\frac{k}{2} N(q) a(q)-\frac{4 \pi G}{3} \varepsilon(a(q)) a^{3}(q) N(q) .
$$

The standard procedure of quantization $\left(p_{q} \rightarrow-i \frac{d}{d q}\right)$ gives the Wheeler-DeWitt equation *

$$
\left(-\frac{d^{2}}{d q^{2}}+V(q)\right) \Psi(q)=0
$$

where

$$
V(q)=\frac{1}{l_{P l}^{4}}\left(N(a(q)) k a(q)-\frac{8 \pi G}{3} \varepsilon(a(q)) a^{3}(q) N(q)\right)
$$

The energy density $\varepsilon(a)$ can be written in the form [12]

$$
\varepsilon=\sum_{n} B_{n} a^{-n}
$$

The coefficients $B_{n}$ refer to contributions of different noninteracting components of the matter content. The parameter $n$ is connected by $n=3(1+\alpha)$ with the parameter $\alpha$ in the equation of state $p=\alpha \varepsilon$.

A choice of the gauge $a^{3-n} N(a)=l_{P l}^{4-n}$ separates a scale-factor-free term in the potential (23), as a result, the Wheeler-DeWitt equation (22) reduces to

$$
\frac{\hbar^{2}}{2 m_{P l}} \frac{d^{2} \Psi}{d q^{2}}-\frac{E_{P l}}{2}(U(a(q))-Q) \psi=0
$$

with the eigenvalue $Q$ given by

$$
Q=\frac{8 \pi}{3} B_{n}
$$

Dependence $q(a)$ is given by

$$
q=\frac{1}{(3-n / 2)} a^{3-n / 2}
$$

\footnotetext{
${ }^{*}$ To allow for an operator ordering the kinetic term should be represented in the form $\frac{1}{q^{p}} \frac{d}{d q} q^{p} \frac{d \Psi}{d q}$. Here we adopt for simplicity $p=0$ following $[8,11]$.
}

Equation (25) describes a quantum system with the quantized quantity $Q$ related to the contribution of the matter component specified by $n$, in the potential $U(q)$ created by other components of the matter content.

An appropriate choice of the boundary conditions is the De Witt boundary condition $\Psi=0$ at $a(q)=0$ [5], and the Vilenkin boundary condition [15] - an outgoing wave function out of a barrier.

Quantization of $\Lambda$ - In the gauge $N(a) a^{3}=1^{\dagger}$ our quantum system is described by the Schrödinger equation (written in the Planckian units)

$$
\frac{d^{2} \Psi}{d q^{2}}-\left(U(q)-Q_{\Lambda}\right) \Psi=0
$$

where

$$
Q_{\Lambda}=\frac{8 \pi}{3} B_{0}=\frac{8 \pi}{3} \varepsilon_{v a c}=\frac{\Lambda}{3} .
$$

Equation (28) describes a quantum system with the quantized quantity $\Lambda$ (vacuum density) in the potential $U(q)$ generated by other matter components which include radiation, dust, and some admixture of strings or quintessence with the equation of state $p=-\varepsilon / 3$. The latter does not affect acceleration but contribute to the curvature term $[16,12,17]$ which facilitates quantum birth of an open and flat universe $[12,13,17]$. In this case

$$
U(a)=\frac{\left(k-B_{s}\right)}{a^{2}}-\frac{B_{d}}{a^{3}}-\frac{B_{\gamma}}{a^{4}} ; \quad a^{3}=3 q
$$

with $B_{s}=\frac{8 \pi}{3} B_{2} ; \quad B_{d}=\frac{8 \pi}{3} B_{3} ; \quad B_{\gamma}=\frac{8 \pi}{3} B_{4}$. In the presence of the curvature term $\left(k-B_{s}\right) / a^{2}$ the potential $U(a(q))$ represents a barrier shown in Fig.1.

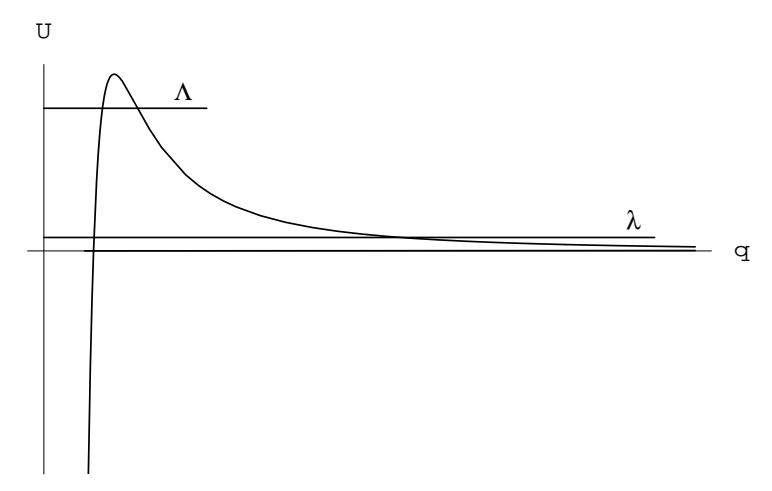

FIG. 1. Typical behavior of a potential $U(q)$. Two relevant (remote past and present) quantum levels of $\Lambda$ are shown.

\footnotetext{
${ }^{\dagger}$ At the classical level this gauge corresponds to such a theory in which $\Lambda$ appears as a constant of integration in the Einstein equations without cosmological term [4].
} 
The potential $U(q)$ has one zero, one maximum, goes to minus infinity as $q \rightarrow 0$, and vanishes at plus infinity, so that negative eigenvalues of $\Lambda$ are confined.

The wave function is the superposition of states

$$
\Psi=\sum_{n} a_{n} \psi_{n}
$$

where the eigenfunctions satisfy

$$
\frac{d^{2} \psi_{n}}{d q^{2}}-\left(U(q)-\lambda_{n}\right) \psi_{n}=0
$$

with the eigenvalues

$$
\lambda_{n}=\frac{8 \pi}{3} \varepsilon_{v a c}
$$

The quasiclassical solution to (32) in the region outside of a barrier is given by

$$
\psi_{n}=\frac{1}{\sqrt{\lambda_{n}-U(q)}} e^{ \pm i \int \sqrt{\lambda_{n}-U(q)} d q}
$$

Qualitative behavior of the probability near the maximum of the potential $U_{m}=U\left(a_{m}\right)$

$$
\left|\psi_{n}\right|^{2} \simeq \frac{1}{\lambda_{n}-U_{m}+\left|U^{\prime \prime}\left(a_{m}\right)\right|\left(a-a_{m}\right)^{2} / 2}
$$

corresponds to domination of a big eigenvalue $\lambda_{n}$.

Behavior far from the maximum of the potential is

$$
\left|\psi_{n}\right|^{2} \simeq \frac{a^{4}}{\lambda_{n} a^{4}-\left(k-B_{s}\right) a^{2}+B_{d} a+B_{\gamma}}
$$

and the probability is maximal near an intersection point $\lambda_{n}=U(q)$ where some $\lambda_{n}$ starts to dominate at some vacuum-dominated stage.

The picture of $\Lambda$ dynamics looks as follows. Making a measurement of $\lambda_{n}$ today, we find its small value $\lambda$ with the big probability. At the beginning of evolution, the biggest probability corresponds to the biggest value $\Lambda$. Evolution involving transitions between quantum levels $\lambda_{n}$ can be related to several scales for symmetry breaking.

Discussion- The aim of this Letter was to note the existence in principle of the possibility to adapt a cosmological constant for description of a dark energy, rather than to give precise estimates in the detailed model.

As we have seen in the framework of the minisuperspace model, the gauge non-invariance of quantum cosmology provides the possibility for quantization of different physical quantities, in particular, of cosmological constant $\Lambda$ associated with the vacuum density $\rho_{v a c}$.

Such a possibility implies that a potential in the Wheeler-DeWitt equation represents a finite barrier. In the case of quantized $\Lambda$ this suggests the presence in the matter content a component with the equation of state $p=-\varepsilon / 3$ which could be some kind of Q-matter, e.g., strings with the negative deficit angle [13].

Indeed, the parameter $k-B_{s}$ at the start of the present vacuum-dominated epoch with $\lambda_{n}=\lambda$ can be estimated at the maximum of the probability (36) as

$$
k-B_{s} \simeq \lambda a^{2}+\frac{B_{d}}{a}
$$

where $\lambda$ corresponds to the vacuum density today $\rho_{v a c} \simeq$ $0.7 \rho_{\text {tot }}[1]$. According to observations, cosmological vacuum responsible for the present inflation starts to dominate at the age $2 \times 10^{9}$ years [18]. The rough estimate of $\rho_{s}$ from (37) suggests, for the case of the vacuumdominated spatially flat universe $(k=0)$, an admixture of a matter component with the equation of state $p=-\varepsilon / 3$, at the level $\rho_{s} \sim 10^{-2} \rho_{\text {tot }}$.

\section{Acknowledgement}

This work was supported by the Polish Ministry of Science and Information Society Technologies through the grant 1P03D.023.27.

[1] N.A. Bahcall et al., Science 284 (1999) 1481; D.N. Spergel et al. (2003) astro-ph/0302209; S.J. Perlmutter et al., Astrophys. J 517 (1999) 565, (2004) astroph/0303428; Tonry J.L. et al.2003, Astrophys. J. 594, 1 (2003); astro-ph/0305008.

[2] J. Ellis (2003) astro-ph/0304183.

[3] B.A. Bassett B.A. et al. 2002, MNRAS 336 (2002) 1217; P.S. Corasaniti and E.J. Copeland, Phys. Rev. D 65 (2002) 043004; S. Hannestad and E. Mortsell 2002, Phys. Rev. D 66 (2002) 063508; K. Takahashi et al. (2003) astro-ph/0305260.

[4] S. Weinberg, Rev. Mod. Phys. 61, 1 (1989).

[5] B. DeWitt (1967), Phys. Rev. 160, 1113; 162, 1239.

[6] J.A. Wheeler, in Battelle Rencontres, Eds. C. DeWitt, J. A. Wheeler, N. Y. Benjamin (1968) 242.

[7] J.J. Halliwell, Phys. Rev. D 38 (1988) 2468.

[8] A. Vilenkin, Phys. Rev. D50 (1994) 2581.

[9] V.A.Savchenko, T.P. Shestakova, G.M. Vereshkov, Int. J. Mod. Phys. A 14 (1999) 4473; T.P. Shestakova, Grav. \& Cosmology 6 Suppl (2000) 47.

[10] M.I. Kalinin, V.N. Melnikov, VNIIFTRI 16(1972) 43.

[11] A. Vilenkin, Phys. Lett. B 117 (1982) 25.

[12] M.L. Fil'chenkov, Phys. Lett. B354 (1995) 208.

[13] I. Dymnikova, M. Fil'chenkov, Phys. Lett. B 545 (2002) 214.

[14] Remarks quoted in: M. Gell-Mann, Phys. Scripta 15 (1987) 202.

[15] A. Vilenkin, Phys. Rev. D 30 (1984) 509.

[16] N.S. Kardashev, Sov. Astron. Zh. 63 (1982) 839.

[17] I.G. Dymnikova, A. Dobosz, M.L. Filchenkov, A. Gromov, Phys. Lett. B 506 (2001) 351.

[18] U. Seljak et al. Phys. Rev. D 71 (2005) 103515. 